doi:10.13108/2021-13-2-99

\title{
INTEGRABLE ABEL EQUATION AND ASYMPTOTICS OF SYMMETRY SOLUTIONS OF KORTEWEG-DE VRIES EQUATION
}

\author{
B.I. SULEIMANOV, A.M. SHAVLUKOV
}

\begin{abstract}
We provide a general solution to a first order ordinary differential equation with a rational right-hand side, which arises in constructing asymptotics for large time of simultaneous solutions of the Korteweg-de Vries equation and the stationary part of its higher non-autonomous symmetry. This symmetry is determined by a linear combination of the first higher autonomous symmetry of the Korteweg-de Vries equation and of its classical Galileo symmetry. This general solution depends on an arbitrary parameter. By the implicit function theorem, locally it is determined by the first integral explicitly written in terms of hypergeometric functions. A particular case of the general solution defines self-similar solutions of the Whitham equations, found earlier by G.V. Potemin in 1988. In the well-known works by A.V. Gurevich and L.P. Pitaevsky in early 1970s, it was established that these solutions of the Whitham equations describe the origination in the leading term of non-damping oscillating waves in a wide range of problems with a small dispersion. The result of this work supports once again an empirical law saying that under various passages to the limits, integrable equations can produce only integrable, in certain sense, equations. We propose a general conjecture: integrable ordinary differential equations similar to that considered in the present paper should also arise in describing the asymptotics at large times for other symmetry solutions to evolution equations admitting the application of the inverse scattering transform method.
\end{abstract}

Keywords: integrability, Abel equation, Korteweg-de Vries equation, asymptotics

Mathematics Subject Classification: 34M55, 35Q53

\section{Passages to the limit in integrable equations}

According to a well-known empirical law, when describing the asymptotics of solutions to integrable differential equations, as a result of reasonable passage to the limit, there can arise only solutions of integrable equations, but often in another sense different from the sense of integrability of original equations.

For instance, all possible continuous limits of such integrable differential-difference equations as Volterra chain

$$
\left(c_{n}\right)_{t}^{\prime}=c_{n}\left(c_{n+1}-c_{n-1}\right)
$$

and Toda chain

$$
\left(z_{n}\right)_{t t}^{\prime \prime}=e^{z_{n+1}-z_{n}}-e^{z_{n+1}-z_{n}}
$$

can be only differential equations integrable in some sense. The same concerns continuous limits of integrable chain discrete in two independent variables. At present, some of this limiting passages are rigorously justified [1].

Remark 1.1. The scientific achievements of R.I. Yamilov made known first of all owing to his papers devoted to various aspects of the integrability of chains of such kind. In his articles [2]-[5], in particular, specific examples of transitions from integrable chains to their continual limits were given.

B.I. Suleimanov, A.M. Shavlukov, Integrable Abel equation and asymptotics of symmetry solutions of Korteweg-De VRies equation.

(C) Suleimanov B.I., Shavlukov A.M. 2021.

Submitted April 1, 2021. 
One of the simplest examples of the passage from one integrable equation to another is the transition from the Korteweg-de Vries (KdV) equation with a small dispersion integrable by the inverse scattering transform method (IST) $(\varepsilon \rightarrow 0)$

$$
u_{t}^{\prime}+u u_{x}^{\prime}+\varepsilon^{2} u_{x x x}^{\prime \prime \prime}=0
$$

to the Hopf equation

$$
u_{t}^{\prime}+u u_{x}^{\prime}=0,
$$

the general solution of which is locally given by the formula

$$
x-t u=F(u) .
$$

The same Hopf equation is a nondissipative limit of the Burgers equation as $\varepsilon \rightarrow 0$ :

$$
u_{t}^{\prime}+u u_{x}^{\prime}=\varepsilon^{2} u_{x x}^{\prime \prime}
$$

This equation is reduced to the heat equation $\Lambda_{t}^{\prime}=\varepsilon^{2} \Lambda_{x x}^{\prime \prime}$ by a linearizing Cole-Hopf transform $u=-2 \varepsilon^{2} \Lambda_{x}^{\prime} / \Lambda$.

A quasiclassical approximation of solutions to an integrable by IST nonlinear Schrödinger equation (NLS) with a small dispersion

$$
-i \varepsilon q_{t}^{\prime}=\varepsilon^{2} q_{x x}^{\prime \prime}+2 \delta|q|^{2} q=0 \quad(\varepsilon \ll 1)
$$

provides a more complicated example of such passage: the substitution

$$
q=\sqrt{h} \exp \left(i \frac{\varphi}{\varepsilon}\right)
$$

reduces (1.1) to a system of two real evolution equations

$$
h_{t}^{\prime}+2\left(h \varphi_{x}^{\prime}\right)_{x}^{\prime}=0, \quad \varphi_{t}^{\prime}+\left(\varphi_{x}^{\prime}\right)^{2}-2 \delta h=\varepsilon^{2} \frac{(\sqrt{h})_{x x}^{\prime \prime}}{\sqrt{h}},
$$

and their dispersionless limit is the system

$$
h_{t}^{\prime}+2\left(h \varphi_{x}^{\prime}\right)_{x}^{\prime}=0, \quad \varphi_{t}^{\prime}+\left(\varphi_{x}^{\prime}\right)^{2}-2 \delta h=0 .
$$

After differentiating the second equation in system $(1.2)$ with respect to the variable $x$ and the change $v=2 \varphi_{x}^{\prime}$, this dispersionless limit becomes a classical hydrodynamical system

$$
h_{t}^{\prime}+(h v)_{x}^{\prime}=0, \quad v_{t}^{\prime}+v v_{x}^{\prime}-4 \delta h_{x}^{\prime}=0 ;
$$

the sign of the constant $\delta$ defines its hyperbolic and elliptic version. Under the hodograph transformation

$$
v(t, x), h(t, x) \rightarrow t(h, v), x(h, v),
$$

the solutions of this system are expressed via the solutions of the linear system of equations

$$
x_{h}^{\prime}=v t_{h}^{\prime}+4 \delta t_{v}^{\prime}, \quad x_{v}^{\prime}=v t_{v}^{\prime}-h t_{h}^{\prime} .
$$

And a very curious transformation of the meaning of integrability occurs [6]-[9] when passing to dispersionless limits of spatially multidimensional equations integrable by IST of the type, for example, the Kadomtsev-Petviashvili equation

$$
\frac{\partial}{\partial t}\left(u_{t}^{\prime}+u u_{x}^{\prime}+\varepsilon^{2} u_{x x x}^{\prime \prime \prime}\right)=u_{y y}^{\prime \prime} .
$$

The integrability [10] of Whitham's hydrodynamic equations by the generalized hodograph method of S.P. Tsarev [11], [12], obtained as a result of averaging integrable ISM equations of the KdV, NLS and sine-Gordon type, is also one of the confirmations of the empirical law formulated in the beginning of the paper.

For example, using this method in combination with the algebraic-geometric method of I.M. Krichever [13], G.V. Potemin [14], self-similar solutions of the Whitham equations were found in explicit form, which, according to the well-known results of A.V. Gurevich and L.P. Pitaevskii [15], 
[16] determine, in the leading order, the behavior as $t \rightarrow \infty$ of a universal special solution of the $\mathrm{KdV}$ equation

$$
u_{t}^{\prime}+u u_{x}^{\prime}+u_{x x x}^{\prime \prime \prime}=0,
$$

with the asymptotics $u=-x^{\frac{1}{3}}+o(1)$ as $x \rightarrow \pm \infty$.

Remark 1.2. In [16], A.V. Gurevich and L.P. Pitaevsky expressed an opinion that the system of ODEs determining these self-similar solutions of Whitham equations cannot be solved explicitly.

Ordinary differential equations (ODEs) of Painlevé type integrable by the isomonodromic deformations method [17, often arising in describing the asymptotics at large times for integrable by IST equations, are also examples of such limiting equations.

Remark 1.3. Among the numerous important scientific results of A.B. Shabat, there is also the following one: when describing the asymptotics at large times of a general initial value problem for the KdV equation (1.3) in [18] and in Section 8.3 of his Habilitation thesis [19], he gave the first example of such transition.

Along with the Painlevé equations themselves, in nonlinear problems with a small parameter including those described by solutions of integrable equations, their higher isomonodromic analogs play a universal role in the description of various contrast transition regimes. Both the Painlevé equations and their higher analogs can be regarded as a kind of nonlinear special functions of wave catastrophes.

The general theory of such nonlinear special functions, the foundations of which were laid in the work of A.V. Kitaev [20] (simultaneously and independently, one particular case, a higher analogue of the second Painlevé equation, was studied by the first of the authors of this work in 21]) is actively developed today and it finds numerous applications [22]-[50].

In particular, while describing the influence of a small dispersion in the vicinity of the cusp points of solutions to dispersionless equations, as a leading term in the asymptotics for the perturbed solution, one uses a special universal solution of the Gurevich-Pitaevsky KdV equation (1.3), which was discussed in the paragraph above Remark 1.2. And as it was stated in [24], [26], this special solution at the same time satisfies ODE

$$
u_{x x x x}^{\prime \prime \prime \prime}+\frac{5 u u_{x x}^{\prime \prime}}{3}+\frac{5\left(u_{x}^{\prime}\right)^{2}}{6}+\frac{5\left(u^{3}-t u+x\right)}{18}=0,
$$

being the first higher representative of in the hierarchy of isomonodromic analogues of the first Painlevé equation in the mentioned paper by A.V. Kitaev [20].

Remark 1.4. The same solution of ODE (1.4) appears in describing of continuous limits of isomonodromic chains [26], [37]. They are related with problems of quantum gravitation theory and were considered in [51], [52].

In [28], V.R. Kudashev observed the following fact: after differentiating (1.4) with respect to $x$, one obtains a stationary part of a non-autonomous higher symmetry of the KdV equation (1.3), which is determined by the linear combination of the stationary parts of its classical Galilean symmetry $u_{\tau_{G}}^{\prime}=1-t u_{x}^{\prime}$ and by its first higher autonomous symmetry

$$
u_{\tau_{5}}^{\prime}=\left(u_{x x x x}^{\prime \prime \prime \prime}+\frac{5 u_{x x}^{\prime \prime} u}{3}+\frac{5\left(u_{x}^{\prime}\right)^{2}}{6}+\frac{5 u^{3}}{18}\right)_{x}^{\prime} .
$$

The symmetry nature of ODE (1.4) and the results of paper [53] allow one to obtain easily the form of aforementioned solution by G.V. Potemin of self-similar Whitham equation [24, [26], 28]. In the last quarter of a century, various properties of solutions to ODE (1.4), and mainly, of course, the special solution of Gurevich-Pitaevsky, have been considered from various points of view in many works, see, for example, [30], [32]-35], [37], [39]-442], [54].

\section{Kudashev integrable Equation}

The asymptotics of the universal Gurevich-Pitaevsky solution as $t \rightarrow \infty$ outside the zone of fast oscillation is given by two roots of the cubic cusp equation:

$$
u^{3}-t u+x=0,
$$


while in the area of non-decaying oscillations it depends on a slow variable $z=x t^{-\frac{3}{2}}$ and fast phase

$$
\Phi=t^{\frac{7}{4}} f(z)+f_{0}(z)
$$

and it reads as

$$
u=\sqrt{t}\left(v_{0}(z, \Phi)+t^{-\frac{7}{4}} v_{1}(z, \Phi)+t^{-\frac{7}{2}} v_{2}(z, \Phi)+\ldots\right) .
$$

This is almost obviously implied by the form

$$
u=\sqrt{t} v(z)
$$

of the solution to cusp equation (2.1) and the form of linearizations by (2.4) of KdV equation (1.3) and ODE (1.4).

In the end of the last century, V.R. Kudashev tried, without employing Whitham averaging method, simply to look for a simultaneous asymptotic solution of form (2.3) to equations (1.3) and (1.4). While doing this, he discovered that the function $f(z)$ determining the leading term of the fast phase 2.2 ) by the formula

$$
R(z)=\frac{7 f(z)}{4 f_{z}^{\prime}}-\frac{3 z}{2}
$$

is related with a solution of a rather simple first order ODE

$$
R_{z}^{\prime}=\frac{486 R^{4}-171 R^{2}+9 z R+5}{9\left(54 R^{3}-9 R+z\right)(2 R+3 z)}
$$

This equation is obviously equivalent to the Abel equation of the second kind:

$$
\left(486 R^{4}-171 R^{2}+5+9 R z\right) z_{R}^{\prime}=972 R^{4}-162 R^{2}+\left(1458 R^{3}-225 R\right) z+27 z^{2} .
$$

Remark 2.1. In terms of solutions of equation 2.5), it is easy to describe the solution of a more general $O D E$

$$
r_{x}^{\prime}=\frac{486 r^{4}-171 t r^{2}+9 x r+5 t^{2}}{9\left(54 r^{3}-9 t r+x\right)(2 t r+3 x)}
$$

Indeed, as $t=0, O D E$ 2.7) is reduced to $O D E r_{x}^{\prime}=r /(3 x)$ with the general solution $r=$ const $\cdot x^{\frac{1}{3}}$. As $t \neq 0$, the solutions of (2.5) and (2.7) are related by the dilatations

$$
r(t, x)=t^{\frac{1}{2}} R(z), \quad z=x t^{-\frac{3}{2}} .
$$

In [37, there were written formulae according to which in the case of a special Gurevich - Pitaevsky solution, the solution $R(z)$ of the ODE (2.5) is related to the self-similar solutions of the Whitham equations from the paper by G.V. Potemin [14]. V.R. Kudashev knew these formulae but never published them during his lifetime.

However, after obtaining V.R. Kudashev equations (2.6) a natural question immediately arose: according to the heuristic law outlined in Section 1 of this paper, this equation must undoubtedly be integrable. But how? Below we provide an answer to this question found last year by using the computer algebra system Maple.

Kudashev equation (2.6) turns out to possess the first integral, which is written explicitly in terms of hypergeometric functions ${ }_{2} F_{1}(\alpha, \beta ; \gamma ; y) \equiv F(\alpha, \beta ; \gamma ; y)$. This integral reads as ( $I$ is an arbitrary constant)

$$
I=\frac{997920 i \sqrt{15}\left(F\left(-\frac{7}{6},-\frac{1}{6} ; \frac{2}{3} ; c_{1}\right)\left(a_{1} b_{6}+b_{8}\right)+\frac{12789 F\left(-\frac{1}{6}, \frac{5}{6} ; \frac{5}{3} ; c_{1}\right) a_{1} c_{2}}{220}\right)}{c_{1}^{\frac{1}{3}}\left(F\left(-\frac{5}{6}, \frac{1}{6} ; \frac{4}{3} ; c_{1}\right) b_{7}+77711400 F\left(\frac{1}{6}, \frac{7}{6} ; \frac{7}{3} ; c_{1}\right) a_{1}\left(b_{5}+b_{9}\right)\right)}
$$


where the functions $a_{i}, b_{i}, c_{i}$ are given by the formulae

$$
\begin{aligned}
& a_{1}(R)=R^{2}-\frac{1}{3}, a_{2}(R)=R^{4}-\frac{25}{111} R^{2}+\frac{1}{1332}, a_{3}(R, z)=R^{3}-\frac{2 R}{7}+\frac{z}{14}, \\
& a_{4}(R)=R^{6}-\frac{55}{174} R^{4}+\frac{19}{1044} R^{2}-\frac{1}{6264}, a_{5}(R)=R^{6}-\frac{503}{3198} R^{4}+\frac{25}{6396} R^{2}-\frac{1}{115128}, \\
& a_{6}(R)=R^{2}\left(3 R^{2}-1\right), a_{7}(R)=-\frac{2665}{1421} R^{6}+\frac{2515}{8526} R^{4}-\frac{125}{17052} R^{2}+\frac{5}{306936}, \\
& a_{8}(R)=\sqrt{3 R^{4}-R^{2}}, a_{9}(R)=R^{2}-\frac{1}{24}, a_{10}(R)=660 R^{4}-76 R^{2}+1, \\
& a_{11}(R)=1980 R^{6}-888 R^{4}+79 R^{2}-1, \\
& b_{1}(R, z)=2 i \sqrt{15} a_{8}(R)\left(3 R^{2}-1\right)+126 R^{4}+9 z R-36 R^{2}, \\
& b_{2}(R)=\sqrt{21}\left(31968 R^{6}-8532 R^{4}+324 R^{2}-1\right)+a_{8}(R)\left(-83160 R^{4}+9576 R^{2}-126\right), \\
& b_{3}(R)=-\frac{148 \sqrt{21} a_{2}(R) a_{8}(R) a_{9}(R)}{1155}+R^{8}-\frac{74}{165} R^{6}+\frac{79 R^{4}-R^{2}}{1980}, \\
& b_{4}(R)=-\frac{533 i \sqrt{35} a_{1}(R) a_{5}(R)}{1421}+5 a_{4}(R) R^{2}, b_{5}(R)=a_{1}(R) R^{2}\left(3 i \sqrt{35} a_{5}(R)+\frac{4263 a_{4}(R)}{533}\right), \\
& b_{6}(R)=\frac{1}{220}\left(i \sqrt{35} a_{6}(R)^{\frac{3}{2}} a_{10}(R)+19188 \sqrt{21} a_{3}(R) a_{5}(R) R-7308 i \sqrt{15} a_{1}(R) a_{4}(R) R^{2}\right), \\
& b_{7}(R, z)=15120 i \sqrt{15} a_{6}(R)^{\frac{3}{2}} a_{11}(R)-124338240 R\left(i \sqrt { 1 5 } a _ { 1 } ( R ) R \left(\sqrt{21} a_{1}(R) a_{5}(R)\right.\right. \\
& \left.\left.\quad-\frac{1036 a_{2}(R) a_{8}(R) a_{9}(R)}{533}\right)-\frac{29841 a_{3}(R, z) a_{4}(R) R^{2}}{533}+7 \sqrt{21} a_{3}(R) a_{5}(R) a_{8}(R)\right), \\
& c_{2}(R)=a_{8}(R) b_{4}(R)+a_{1}(R) R^{2}\left(\sqrt{21} a_{7}(R)+i \sqrt{15} a_{4}(R)\right) \cdot \\
& \left.c_{1}(R, z)=-\frac{2984 a_{3}(R, z) a_{4}(R) R}{148}\right), \\
& b_{8}(R, z)=\frac{444 a_{8}(R)}{385}\left(3 i \sqrt{35} a_{1}(R)^{2} a_{2}(R) a_{9}(R)-\frac{298}{53}(R, z) b_{2}(R) b_{3}(R)\right. \\
& b_{9}(R)=a_{8}(R)\left(-\sqrt{21} a_{1}(R) a_{5}(R)-\frac{1421 i \sqrt{15} a_{4}(R) R^{2}}{15} a_{1}(R),\right.
\end{aligned}
$$

In the following table we provide approximate values of $I$ corresponding to solutions of five different initial value problems for ODE (2.6).

\begin{tabular}{|l|l|l|}
\hline Initial value & Approximate value of $I$ & Interval of calculation in $R$ \\
\hline$z(0)=0$ & $0.0960605+0.1663816 i$ & $R \in[-10,10]$ \\
\hline$z(1)=0$ & $0.0194046+0.0336097 i$ & $R \in[-11,11]$ \\
\hline$z(5)=7$ & $0.0308202+0.0533821 i$ & $R \in[-1,15]$ \\
\hline$z(50)=75$ & $0.03177193+0.05503056 i$ & $R \in[-1,60]$ \\
\hline$z(-0.57735)=0.3849$ & $0.000591+0.0010237 i$ & $R \in[-10,0]$ \\
\hline
\end{tabular}

Table 1: Approximate values of $I$ corresponding to five different initial value problems.

\section{Conclusion}

In the opinion of the authors of the paper, its result is potentially not only of a particular importance. 
For example, there arises a conjecture that the systems of two non-autonomous ODEs

$$
\begin{aligned}
A_{s}^{\prime} & =\frac{(2 A-1)\left(-288 A^{3}+192 A^{2}+24 s A-27 A-4 s+4 B\right)}{(A+2 s)\left(-576 A^{3}+504 A^{2}-126 A+48 s A+8 B-12 s+9\right)}, \\
B_{s}^{\prime} & =\left(36 A+3 s-54 A^{2}-\frac{9}{2}\right) A_{s}^{\prime}+\frac{108 A^{3}-108 A^{2}-6 s A+6 B+27 A+4 s}{2 A+4 s}, \\
A_{s}^{\prime} & =\frac{(2 A-1)\left(288 A^{3}-192 A^{2}+24 s A+27 A-4 s-4 B\right)}{(A-2 s)\left(-576 A^{3}+504 A^{2}-126 A-48 s A+8 B+12 s+9\right)}, \\
B_{s}^{\prime} & =\left(36 A-3 s-54 A^{2}-\frac{9}{2}\right) A_{s}^{\prime}-\frac{108 A^{3}-108 A^{2}+6 s A+6 B+27 A-4 s}{2 A-4 s},
\end{aligned}
$$

considered in papers by R.N. Garifullin [44, [46] should also possess two first integrals, which can be explicitly written in terms of hypergeometric functions. By means of solutions to systems of ODEs (3.1) and (3.2), one describes the asymptotics as $t \rightarrow \infty$ of form

$$
u=t\left(v_{0}(z, \Phi)+t^{-\frac{5}{4}} v_{1}(z, \Phi)+t^{-\frac{5}{2}} v_{2}(z, \Phi)+\ldots\right), \quad \Phi=t^{\frac{5}{2}} f(s)+n(s) \quad\left(s=\frac{x}{t^{2}}\right)
$$

for simultaneous solutions of $\mathrm{KdV}$ equation (1.3) and fifth order ODE

$$
\left(u_{x x x x}^{\prime \prime \prime \prime}+\frac{5 u_{x x}^{\prime \prime} u}{3}+\frac{5\left(u_{x}^{\prime}\right)^{2}}{6}+\frac{5 u^{3}}{18}\right)_{x}^{\prime} \pm \frac{2 u+x u_{x}^{\prime}-3 t\left(u_{x x x}^{\prime \prime \prime}+u u_{x}^{\prime}\right)}{6}=0,
$$

determined by linear combinations of stationary parts of the first higher non-autonomous symmetry (1.5) and a classical dilatation symmetry $u_{\tau_{r}}=2 u+x u_{x}^{\prime}-3 t\left(u_{x x x}^{\prime \prime \prime}+u u_{x}^{\prime}\right)$.

We propose a conjecture: a similar asymptotics at large times for simultaneous solutions of various nonlinear integrable by IST evolution equations with ODEs of the higher Painlevé type determined by non-autonomous symmetries and, more generally, by invariant manifolds of these evolution equations, should also be described by ODEs integrable in a similar sense.

\section{BIBLIOGRAPHY}

1. L.A. Kalyakin. Asymptotic transitions from discrete to continuous models // Teor. Matem. Fiz. 76:3, 323327 (1988). [Theor. Math. Phys. 76:3, 891-894 (1988).]

2. R.I. Yamilov. On classification of discrete evolution equations // Uspekhi Matem. Nauk. 38:6(234), 155-156 (1983). (in Russian).

3. D. Levi, P. Winternitz, R.I. Yamilov. Symmetries of the continuous and discrete Krichever-Novikov equation // SIGMA. 7, 097 (2011).

4. R.N. Garifullin, R.I. Yamilov. On integrability of a discrete analogue of Kaup-Kupershmidt equation // Ufimskji Matem. Zhurn. 9:3, 158-164 (2017). [Ufa Math. J. 9:3, 158-164 (2017).]

5. R.N. Garifullin, R.I. Yamilov. On the integrability of a lattice equation with two continuum limits // Itogi Nauki Tekh. Ser. Sovrem. Matem. Pril. Temat. Obz. VINITI RAN. 152, 159-164 (2018). [Journal of Mathematical Sciences 252:2, 283-289 (2021)].

6. V.E. Zakharov. Dispersionless limit of integrable systems in $2+1$ dimensions // In: "Singular limits of dispersive waves", Ercolani, N.M. et al. (eds.). Plenum Press, New York, 165-174 (1994).

7. E.V. Ferapontov, K.R. Khusnutdinova. Hydrodynamic reductions of multi- dimensional dispersionless PDEs: the test for integrability // J. Math. Phys. 45:6, 2365-2377 (2004).

8. E.V. Ferapontov, K.R. Khusnutdinova. On integrability of (2+1)-dimensional quasilinear systems // Comm. Math. Phys. 48, 187-206 (2004).

9. E.V. Ferapontov, B. Kruglikov. Dispersionless integrable systems in 3D and Einstein-Weyl geometry // J. Diff. Geom. 97, 215-254 (2014).

10. B.A. Dubrovin, S.P. Novikov. Hydrodinamics of weakly deformed soliton lattices. Differential geometry and Hamiltonian theory // Uspekhi Matem. Nauk. 44:6(270), 29-98 (1989). [ Russ. Math. Surv. 44:6, 35-124 (1989).]

11. S.P. Tsarev. On Poisson brackets and one-dimensional Hamiltonian systems of hydrodynamic type // Dokl. AN SSSR. 282:3, 534-537 (1985). [Sov. Math. Dokl. 31, 488-491 (1985).]

12. S.P. Tsarev. The geometry of hamiltonian systems of hydrodynamic type. The generalized hodograph method // Izv. AN SSSR. Ser. Matem. 54:5, 1048-1068 (1990). [Math. USSR-Izv. 37:2, 397-419 (1991).]

13. I.M. Krichever. Method of averaging for two-dimensional "integrable" equations // Funkts. Anal. Pril. 22:3, 37-52 (1988). [Funct. Anal. Appl. 22:3, 200-213 (1988).] 
14. G.V. Potemin. Algebro-geometric construction of self-similar solutions of the Whitham equations // Uspekhi Matem. Nauk. 43:5(263), 211-212 (1988). [Russ. Math. Surv. 43:5, 252-253 (1988).]

15. A.V. Gurevich, L. P. Pitaevskii. Breaking a simple wave in the kinetics of a Rarefied Plasma // Zhurn. Eksper. Teor. Fiz. 60:6, 2155-2174 (1971). [Sov. Phys. JETP. 33:6, 1159-1167 (1971).]

16. A.V. Gurevich, L.P. Pitaevskii. Non stationare structure of collisionless shock wave // Zhurn. Eksper. Teor. Fiz. 65:2, 590-604 (1973). [Sov. Phys. JETP. 38:2, 291-297 (1974).]

17. A Fokas, A.R. Its, A.A. Kapaev, V.Yu. Novokshenov. Painlevé transcendents. The Riemann-Hilbert approach. Regul. Khaot. Dinam., Moscow-Izhevsk (2005). [Amer. Math. Soc., Providence, RI (2006).]

18. A.B. Shabat. On the Korteweg-de Vries equation // Dokl. AN SSSR. 211:6, 1310-1313 (1973). [Sov. Math. Dokl. 14, 1266-1270 (1973).]

19. A.B. Shabat. Transformation operators and nonlinear equations // Habiliation thesis. Bashkir State Univ., Ufa (1974). (in Russian).

20. A.V. Kitaev. Turning points of linear systems and double asymptotics of the Painlevé transcendents // Zap. LOMI 187, 53-74 (1991). [J. Math. Sci. 73:4, 446-459, (1995).]

21. B.I. Suleimanov. The second Painlevé equation in a problem concerning nonlinear effects near caustics // Zap. LOMI. 187, 110-128 (1991). [J. Math. Sci. 73:4, 482-493 (1995).]

22. B.I. Suleimanov. A "nonlinear" generalization of special functions of wave catastrophes described by double integrals // Matem. Zamet. 52:5, 102-106 (1992). [Math. Notes. 52:5,6, 1146-1149 (1992).]

23. B.I. Suleimanov, I.T. Habibullin. Symmetries of the Kadomtsev-Petviashvili equation, the isomonodromic deformations, and "nonlinear" generalizations of special functions of wave catastrophes // Teor. Matem. Fiz. 97:2, 213-216 (1993). [Theor. Math. Phys. 97:2, 1250-1258, (1993).]

24. B.I. Suleimanov.Solution of the Korteweg - de Vries equation which arises near the breaking point in problems with a slight dispersion // Pisma ZhETF. 58:11, 606-610 (1993). [JETP Lett. 58:11, 849-854 (1993).]

25. B.I. Suleimanov. Influence of a weak nonlinearity on the high-frequency asymptotics in caustic rearrangements // Teor. Matem. Fiz. 98:2, 198-206 (1994). [Theor. Math. Phys. 98:2, 132-138 (1994).]

26. B.I. Suleimanov. Onset of nondissipative shock waves and the "nonperturbative" quantum theory of gravitattion // Zhurn. Eskper. Teor. Fiz. 105:5, 1089-1099 (1994). [J. Exper.Theor. Phys. 78:5, 583-587 (1994).]

27. A.V. Kitaev. Caustics in $1+1$ integrable systems // J. Math. Phys. 35:2, 2934-2954 (1994).

28. V.R. Kudashev. KdV shock-like waves as invariant solutions of KdV equaton symmetry // Preprint: arXiv preprint patt-sol/9404002. (1994).

29. V.R. Kudashev, B.I. Suleimanov. Characteristic features of some typical spontaneous intensivity collapse processes in unstable media // Pisma ZhETF. 62:4, 358-362 (1995). [JETP Lett. 62:4, 382-388, (1995).]

30. V. Kudashev, B. Suleimanov. A soft mechanism for generation the dissipationless shock waves // Phys. Lett. A. 221:3, 4, 204-208 (1996). 1996.

31. V.R. Kudashev, B.I. Suleimanov. Small-amplitude dispersion oscillations on the background of the nonlinear geometric optics approximation // Teor. Matem. Fiz. 118:3, 413-422 (1999). [Theor. Math. Phys. 118:3, $325-332$ (1999).]

32. V.R. Kudashev, B.I. Suleimanov. The effect of small dissipation on the onset of one-dimensional shock waves // Prikl. Matem. Mekh. 65:3, 456-466 (2001). [J. Appl. Math. Mech. 65:3, 441-451 (2001).]

33. B. Dubrovin. On Hamiltonian perturbations of hyperbolic systems of conservation laws, II: Universality of critical behaviour // Comm. Math. Phys. 267, 117-139 (2006).

34. T. Claeys, M. Vanlessen. The existence of a real pole-free solution of the fourth order analogue of the Painlevé I equation // Nonlinearity. 20:5, 1163-1184 (2007).

35. T. Grava, C. Klein. Numerical study of a multiscale expansion of the Korteweg-de Vries equation and Painlevé-II equation // Proc. Royal Society A. 464:2091, 733-757 (2008).

36. B. Dubrovin, T. Grava, C. Klein. On Universality of Critical Behavior in the Focusing Nonlinear Schrödinger Equation, Elliptic Umbilic Catastrophe and the Tritronquée Solution to the Painlevé-I Equation // J. Nonl. Sci. 19:1, 57-94 (2009).

37. R. Garifullin, B. Suleimanov, N. Tarkhanov. Phase Shift in the Whitham Zone for the Gurevich-Pitaevskii Special Solution of the Korteweg-de Vries Equation // Phys. Lett. A. 374:13, 14, 1420-1424 (2010). 2010.

38. R.N. Garifullin, B.I. Suleimanov. From weak discontinues to nondissipative shock waves // Zh. Eksp. Teor. Fiz. 137:1, 149-165 (2010). [J. Exper. Theor. Phys. 110:1, 133-146 (2010).]

39. T. Claeys. Asymptotics for a special solutions to the second member of the Painleve I hierarhy // J. Phys. A. 43:43, 434012. 18 pp. (2010).

40. T. Claeys, T. Grava. Solitonic asymptotics for the Korteweg-de Vries equation in the small dispersion limit // SIAM J. Math. Anal. 42:5, 2132-2154 (2010).

41. T. Claeys. Pole-free solutions of the first Painlevé Hierarchy and non-generic critical behavior for the KdV equation // Physica D: Nonl. Phen. 241:23, 2226-2236 (2011). 
42. T. Grava, A. Kapaev, C. Klein. On the Tritronquée Solutions of $P_{I}^{2} / /$ Constr. Approx. 41:3, 425-466 (2015).

43. B. Dubrovin, M. Elaeva. On the critical behavior in nonlinear evolutionary PDEs with small viscosity // Russ. J. Math. Phys. 19:4, 449-460 (2012).

44. R.N. Garifullin. Phase shift for the common solution of KdV and fifth order differential equation // Ufimskij Matem. Zhurn. 4:2, 80-86 (2012). [Ufa Math. J. 4:2, 52-61 (2012).]

45. M. Bertola, A. Tovbis. Universality for the Focusing Nonlinear Schrödinger Equation at the Gradient Catastrophe Point: Rational Breathers and Poles of the Tritronquée Solution to Painlevé I // Comm. Pure and Appl. Math. 66, 678-752 (2013).

46. R.N. Garifullin. On simultaneous solution of the KdV equation and a fifth-order differential equation // Ufimskij Matem. Zhurn. 8:4, 53-62 (2016). [Ufa Math. J. 8:4, 80-86 (2016).]

47. B. I. Suleimanov. Effect of a small dispersion on self-focusing in a spatially one-dimensional case // Pisma ZhETF. 106:6, 375-380 (2017). [JETP Lett. 6:6, 400-405 (2017).]

48. B.I. Suleimanov. On analogs of wave catastrophe functions that are solutions of nonlinear integrable equations // Itogi Nauki Tekh. Ser. Sovrem. Matem. Pril. Temat. Obz. VINITI RAN. 163, 81-95 (2019). (in Russian).

49. D. Bilman, L. Ling, P.D. Miller. Extreme superposition: rogue waves of infinite order and the Painlevé-III hierarchy // Duke Math. J. 169, 671-760 (2020).

50. V.E. Adler. Nonautonomous symmetries of the KdV equation and step-like solutions// J. of Nonlinear Math. Phys. 27:3, 478-493 (2020).

51. E. Bresin, E. Marinari, G. Parisi. A nonperturbative ambiguty free solution of a string model // Phys. Lett. B. 242:1, 35-38 (1990).

52. M. Douglas, N. Seiberg, S. Shenker. Flow and unstability in quantum gravity // Phys. Lett. B. 244:3,4, 381-386 (1990).

53. V.R. Kudashev, S.E. Sharapov. Inheritance of KdV symmetries under Whitham averaging and hydrodynamic symmetries of the Witham equations // Teor. Matem. Fiz. 87:1, 40-47 (1991). [Theor. Math. Phys. 87:1, 358-363 (1991).]

54. B.I. Suleimanov. "Quantizations" of higher Hamiltonian analogues of the Painlevé I and Painlevé II equations with two degrees of freedom // Funkts. Anal. Pril. 48:3, 52-62 (2014). [Funct. Anal.Appl. 48:3, 198-207 (2014).]

Bulat Irekovich Suleimanov, Institute of Mathematics,

Ufa Federal Research Center, RAS,

Chernyshevky str. 112,

450008, Ufa, Russia

E-mail: bisul@mail.ru

Azamat Mavletovich Shavlukov,

Institute of Mathematics,

Ufa Federal Research Center, RAS,

Chernyshevky str. 112,

450008, Ufa, Russia

Bashkir State University,

Zaki Validi str. 32,

450076, Ufa, Russia

E-mail: aza3727@yandex.ru 